\title{
Adsorption of Cupric Ion in Wastewater with Natural Oxidized Celluloses
}

\author{
Geng Cunzhen ${ }^{1,2, a}$, Quan Fengyu ${ }^{1,2, b}, X_{1 a}$ Yanzhi $^{2,3, \mathrm{c}}$,Wang Weijun ${ }^{1, \mathrm{~d}}$, Zhao Zhihui ${ }^{2,3, \mathrm{e}}$, Yang Manli ${ }^{4, \mathrm{f}}$, Zhao \\ Xihui $^{1,2, \mathrm{~g}}$ \\ ${ }^{1}$ College of Environmental Science and Engineering, Qingdao University, Qingdao, 266071, Shandong, PR China. \\ ${ }^{2}$ Co-Innovation Center for Marine Biomass Fibers, Materials and Textiles of Shandong Province, Qingdao, 266071, \\ Shandong, PR China. \\ ${ }^{3}$ Cultivating Base of National Key Lab of Novel Fibers and Modern Textiles Qingdao University, Qingdao, 266071, \\ Shandong, PR China. \\ ${ }^{4}$ College of Chemistry and Pharmacy, Qingdao Agricultural University, Qingdao, 266000, Shandong, PR China. \\ E-mail: ${ }^{\mathrm{a}}$ qdugcz@126.com; ${ }^{\mathrm{b}} \mathrm{qfyqdu} @ 163 . c o m ;{ }^{\mathrm{c} x y z h q d u @ 163 . c o m ;}$; 494382656@qq.com; ${ }^{\mathrm{e}}$ zzh_0904@163.com; \\ gaozxh@163.com; ${ }^{\mathrm{f}}$ mlyang@163.com \\ Corresponding author:Xia Yanzhi, e-mail: xyzhqdu@163.com.
}

\begin{abstract}
The aim of this paper was to investigate adsorption of cupric ionin wastewater with a new type of adsorbent, namely natural oxidized celluloses(NOCs), whichwas synthesized in a specialselective oxidation system withraw cellulose. The characterization of NOCs was investigated by FTIR and the morphology of NOCs was examined with scanning electron microscope. The effects of adsorbent dosage, $\mathrm{pH}$, temperature, initial concentration and adsorption time on the adsorption efficiencywere explored on the adsorption efficiency. The results demonstrated thatthe removal efficiency of $\mathrm{Cu}^{2+}$ could be achieved as high as $99 \%$ under the conditions of the temperature $25^{\circ} \mathrm{C}$, the $\mathrm{Cu}^{2+}$ concentration $100 \mathrm{mg} \cdot \mathrm{l}^{-1}$, $\mathrm{pH} 5 \sim 6$ in solution, dosing 0.175gNOCs with one hour's shaking. Adsorption isothermal studies also revealed that the adsorption process of $\mathrm{Cu}^{2+}$ was well fitted with the Freundlich and Langmuir type adsorption isotherm. In conclusion, because of the raw cellulose is the most abundantbiomasson the earth as well as a renewable and biodegradable material, NOCs may have broad application in wastewater treatment industry as a new adsorption material in the future.
\end{abstract}

Keywords: $\quad \mathrm{Cu}^{2+}$,NOCs, adsorption, influencing factors, wastewater treatment

\section{INTRODUCTION}

Modern industries discharge lots of wastewater containing large amounts of heavy metals. Because of theirs toxic, bioaccumulation and their various forms, these metals do harm to the ecological environment and human survival after entering the environment. Cupric ion is toxic to the human health and ecological environment.

A number of methods are available to remove heavy metals from water or wastewater, including chemical precipitation, ion exchange, adsorption, membrane process, reverse osmosis, and so on[1-6]. Among these technologies, adsorption is considered to be the most effective and efficient approach for dealing with large volumes of wastewater in recent years[7]. Adsorption process has been applied as one of the most popular treatment processes due to various advantages, such as high efficiency, low cost, wide applicable scope, and many others.
Many researchers have carried out studies on the removal of copper from waste water using different adsorbents, such as nanometer-sized $\mathrm{SiO}_{2}$, coal fly ash, chitosan and cellulose hydrogel[8-12]. Cellulose, a kind of inexpensive materials and the main constituent of plants, is the most abundant biodegradable natural polymer biomass on the earth, has been utilized in many applications such as pulp, paper, filters, film, and the textile industries[13]. To use cellulose as a heavy metal ion adsorbent, however, chemical modifications such as esterification, etherification, and oxidation targeting the hydroxyl group present in cellulose are essential[14,15].

In this paper, a new adsorption material, namely natural oxidized celluloses (NOCs) was prepared by the chemical-modified natural cellulose. And copper ion adsorption experiments were also carried out in order to study the adsorption properties of the material. Natural cellulose has great superiority as a new experimental material, since cellulose is the most abundant and renewable biopolymers, and is one of the promising raw materials available in terms of cost for the preparation of various functional materials. Therefore this new type of adsorbent may have potential for application in wastewater treatment. With the single variable controlling method, all kinds of factors affecting the adsorption efficiency were studied and the optimum conditions of the new removal of bivalent copper ion with NOCs were performed.

\section{MATERIALS AND METHODS}

\subsection{Materials}

The new adsorbent (NOCs) was synthesized in our laboratory. The wastewater used in batch experiments was simulated wastewater. Raw natural cellulose used in the present investigation was obtained from Shandong Sun Paper Industry Joint Stock Co., Ltd. Deionized water was used for all experiments. Other reagents used were of analytical grade in all experiments.

\subsection{Synthesis and characterization of NOCs}

The natural cellulose (NC) was oxidized in an 
oxidation system constituted by 2 , 2, 6 , 6-Tetramethylpiperidinooxy (TEMPO) $/ \mathrm{NaBr} / \mathrm{NaClO}$ under certain conditions[16,17]. The new adsorbent is a long chain molecule with lots of hydroxyl and some carboxyl groups, and its structure is illustrated in Fig. 1.

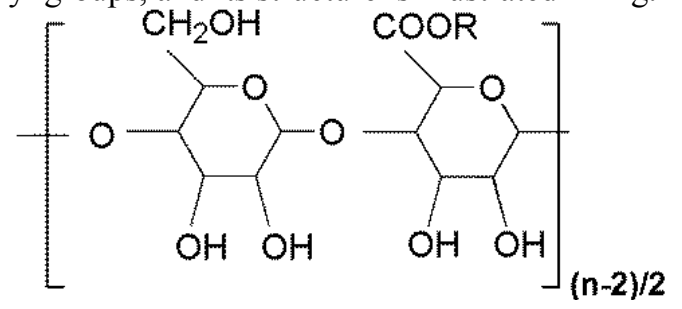

Figure 1.Structure of NOCs (R, H or $\mathrm{Na})$

The detail of the synthesis of NOCs is following: Designed weight $\mathrm{NC}$ was suspended in purified water which contained TEMPO $(0.1 \mathrm{mmol} / \mathrm{g} \mathrm{NC})$ and sodium bromide $(1.0 \mathrm{mmol} / \mathrm{g} \mathrm{NC})$. Then, a designed amount of the $10 \% \mathrm{NaClO}$ solution $(5.0 \sim 10.0 \mathrm{mmol} / \mathrm{g} \mathrm{NC})$ was added to the slurry in dropwise, and the mixture was stirred by mechanical at room temperature. And meanwhile the $\mathrm{pH}$ of the mixture system was maintained between 10.5 and 11.0 using a $\mathrm{pH}$ stat until no $0.5 \mathrm{M}$ $\mathrm{NaOH}$ consumption was observed in the reaction process. The oxidation was quenched by adding ethanol (ca.10 $\mathrm{mL}$ ). The oxidized cellulose was washed thoroughly with purified water and then ethanol by filtration. The wet product was dried by lyophilization then followed by vacuum-drying at $50{ }^{\circ} \mathrm{C}$ for about $48 \mathrm{~h}$.

The production characterization was investigated by FTIR (NICOLET 5700 spectrometer, USA). The morphology of NOCs was examined with scanning electron microscope (SEM,TM3000, Jiangsu vanke science instrument co., LTD, China).

\subsection{Adsorptionexperiments.}

The adsorption was processed in following steps: 1) the volume of $20 \mathrm{ml}$ wastewater of different concentrations were taken into the small beakers; 2) a certain amount adsorption materials were filled into the solution after its $\mathrm{pH}$ was adjusted with sodium hydroxide and hydrochloric acid; 3) all the supernatant solution were put into a constant temperature oscillator for some designed time; 4) The concentration of $\mathrm{Cu}^{2+}$ were determined with sodium diethyldithiocarbamate spectrophotometry after stopping oscillating and standing for a period of time. Therefore, the optimum adsorption conditions were obtained on the basis of setting the adsorption conditions on batch experiments.

\subsection{Calculation method}

The effect of adsorbing $\mathrm{Cu}^{2+}$ can be formulated by adsorbing capacity $\left(\mathrm{q}_{\mathrm{e}}\right)$ and adsorption efficiency ( $\left.\mathrm{R} \%\right)$.

Adsorbing capacity $\left(\mathrm{q}_{\mathrm{e}}\right)$ refers to the quality of $\mathrm{Cu}^{2+}$ adsorbed into the adsorbent (1g mass) when the adsorption system achieves adsorption equilibrium. And it was calculated according to Equation (1):

$$
q_{e}=\frac{\left(C_{0}-C_{e}\right) \times V}{m}
$$

Where $\mathrm{q}_{\mathrm{e}}\left(\mathrm{mg} \mathrm{g}^{-1}\right)$ is the adsorbing capacity, $\mathrm{C}_{\mathrm{o}}\left(\mathrm{mg} \mathrm{l}^{-1}\right)$ is the initial concentration of $\mathrm{Cu}^{2+}, \mathrm{C}_{\mathrm{e}}\left(\mathrm{mg} \mathrm{l}^{-1}\right)$ is the equilibrium concentrations of $\mathrm{Cu}^{2+}, \mathrm{V}$ (l) is the total volume of wastewater and $\mathrm{m}(\mathrm{g})$ is the mess of adsorbent.

The removal efficiency ( $\mathrm{R} \%$ ) refers to specific value between the quality of $\mathrm{Cu}^{2+}$ adsorbed into the adsorbent and the initial quality of $\mathrm{Cu}^{2+}$ when the adsorption system achieves adsorption equilibrium. It is defined as Equation (2):

$$
R \%=\frac{\left(C_{0}-C_{e}\right)}{C_{0}} \times 100 \%
$$

\section{RESULTS AND DISCUSSION}

\subsection{FTIR}

FT-IR spectra of the cellulose and NOCsare shown in Fig. 2. The natural cellulosehas almost no absorption band from 1400 to $1700 \mathrm{~cm}^{-1}$, but two strong absorption bands at 1608 and $1426 \mathrm{~cm}^{-1}$ derived from the carbonyl groups present in response to NOCs, which are used for quantification purposes representing the appearance of $\mathrm{C}=\mathrm{O}$ stretching vibration and $\mathrm{C}-\mathrm{O}$ symmetric stretching vibration in respectively. In addition, a slight reduction of the bands related to hydrogen bonds stretching vibration of $\mathrm{h}$ hydroxyl groups at $3377 \mathrm{~cm}^{-1}$ and to stretching vibrations of $\mathrm{CH}$ at $2901 \mathrm{~cm}^{-1}$ is obviously observed. From these results, it can be concluded that hydroxyl groups at the C6 position of cellulose molecules are converted to sodium carboxylate and NOCs can be successfully prepared.

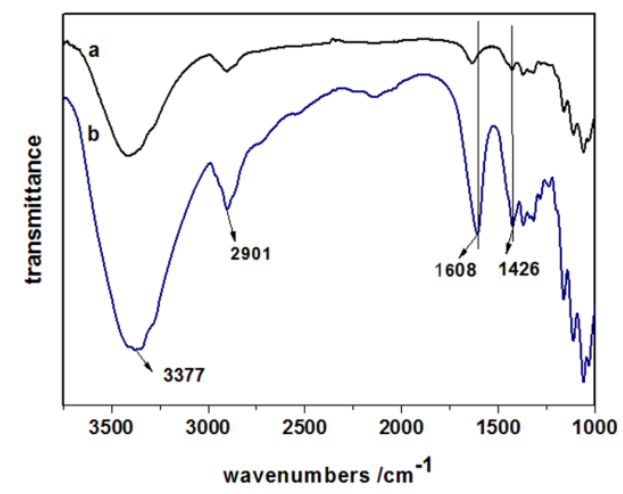

Figure 2.FT-IR spectra of a (natural cellulose) and b (NOCs).

\subsection{SEM}

The morphologies of natural cellulose and NOCs were studied by SEM analysis in order to make a comparison more directly before and after synthesis of NC. Two typical images at two magnifications of NC and NOCs are showed in Fig.3a and b.Evidently, both of them are characterized by a level of inhomogeneity owing to the natural growing. And the dimension of NOCs is $4-8 \mathrm{~nm}$ in width. But there many mangy sags and crests in surface of NOCs, which increase undoubtedly the surface area of the adsorbents.

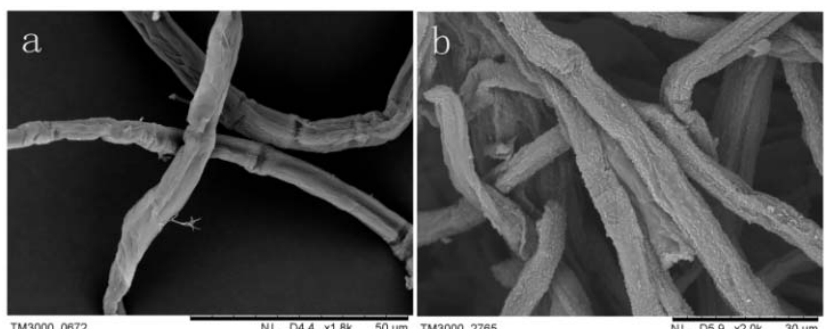

Figure 3.SEM micrographs of a (natural cellulose) and b (NOCs). 


\subsection{Adsorption results}

\subsubsection{Effect of adsorbent dosage}

Fig. 4shows the influence of adsorbent dosage on the removal efficiency of $\mathrm{Cu}^{2+}$. The removal efficiency of $\mathrm{Cu}^{2+}$ increased significantly with the increasing of the amount of adsorbent in range of $0.1 \sim 0.15 \mathrm{~g}$, then reached plateau in the range of $0.15 \sim 0.175 \mathrm{~g}$ of the adsorbent dosage, and the efficiency began to fell slightly when the adsorbent dosage was larger than $0.175 \mathrm{~g}$. It could be inferred that the removal efficiency of $\mathrm{Cu}^{2+}$ was the optimum and adsorption process was saturated when the adsorbent dosage was about $0.175 \mathrm{~g}$.

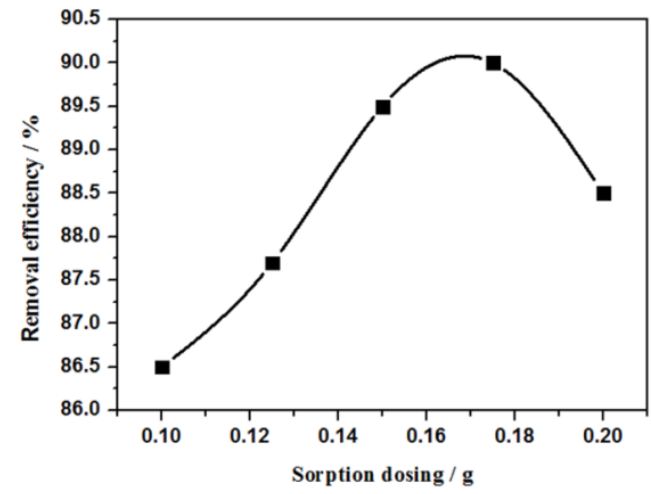

Figure 4.The influence of adsorbent dosage on removal efficiency of $\mathrm{Cu}^{2+}$.

The behavior of the efficiency dependency on the absorbent dosage could be explicated that the removal efficiency of heavy metal increased along with the increase of absorbent quality, i.e. with the increase in the number of active adsorption sites[18]. Moreover, due to uneven distribution of oxidized cellulose surface position, adsorption sites had strong and weak points naturally. The surface active points form competitive adsorption among the adsorption sites when the adsorbent dosage was in excess. In addition, owing to the hydrogen-bond interaction, the cross-linking among the high molecules of the adsorbent would be formed in this case. So it would lead to the result that surface active points can notbe made full use of, and was not conducive to the adsorption. Thereby, it would result in the decrease of the efficiency.

\subsubsection{Effect of adsorption time}

In our research, we found that the adsorption time was one of the important influence factors. Fig. 5 illustrates the influence of adsorption time on removal efficiency of $\mathrm{Cu}^{2+}$. At the beginning, concentration gradient between the solution and the surface of NOCs was very high and hence the driving force was big too, this resulted adsorption speed was very high. The removal efficiency of $\mathrm{Cu}^{2+}$ had reached more than $90 \%$ at the time of $30 \mathrm{~min}$, and then the removal efficiency still increased despite of the concentration gradient decreasing and the adsorbent tending to saturation gradually. From Fig.5, the adsorption efficiency was reaching the maximum and the adsorbent getting to saturation at $60 \mathrm{~min}$. As time going on, desorption began to appear and resulted in the declining of the removalefficiency.

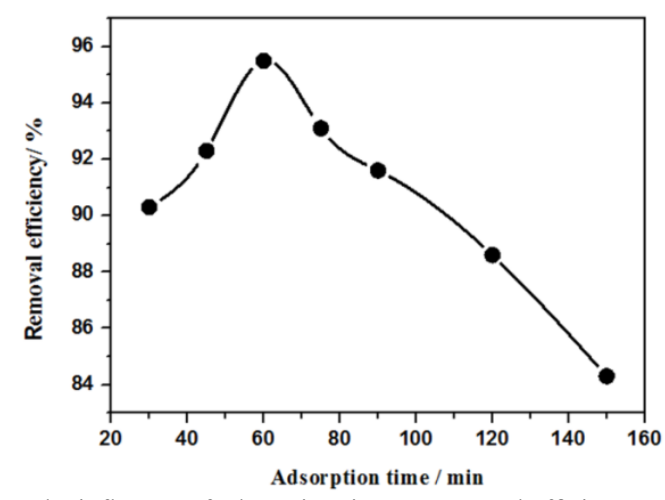

Figure 5.The influence of adsorption time on removal efficiency of $\mathrm{Cu}^{2+}$

\subsubsection{Effect of initial concentration of metal ion}

The initial concentration of metal ions had effect on the occurrence of ion exchange and complexation in the suspended solution. The dependency of the removal efficiency of $\mathrm{Cu}^{2+}$ on the initial concentration of metal ion is shown in Fig.6. Meanwhile, the graph presented a curve rising at first and declining in the studied range, while the best initial concentration of the oxidized cellulose adsorption $\mathrm{Cu}^{2+}$ is $100 \mathrm{mg} \cdot \mathrm{L}^{-1}$. This may be due to the fact that when the initial concentration increased in the process of the adsorption, the concentration gradient increased between the solution and the surface of NOCs, thereby the driving force increased. The adsorption capacity of NOCs increased rapidly at first, and then went back into equilibrium. When it surpassed the equilibrium, the high concentration solution reduced the chance of functional atoms combining with $\mathrm{Cu}^{2+}$, due to the fact that the amount of $\mathrm{Cu}^{2+}$ in the solution exceeded the maximum adsorbing capacity of adsorbent at this time. Therefore, the adsorption quantity no longer increased. So the excessive increase of initial concentration led to reducing of the adsorption efficiency. It showed that the initial concentration had a fitting range.

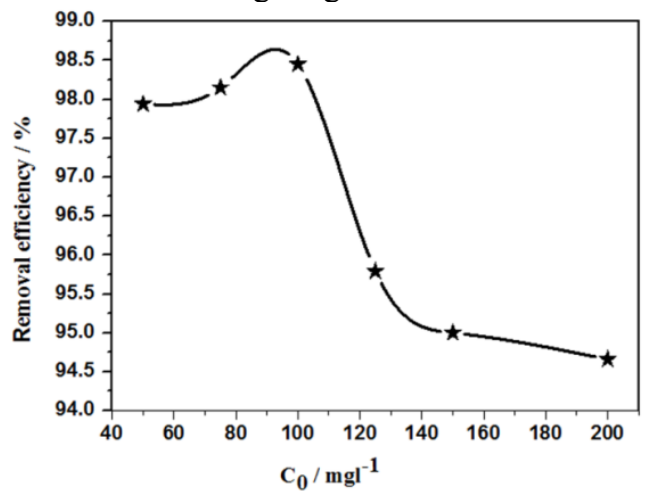

Figure 6.The influence of initial concentration of metal ion on removal efficiency of $\mathrm{Cu}^{2+}$.

\subsubsection{Effect of temperature}

Temperature was one of the critical factors affecting adsorption. It was mainly due to the high sensitivity of the solubility of copper on the temperature in a certain temperature range, which promoted the adsorption. However, when the temperature exceeded a limit, high temperature would lead to excessive thermal motion of the molecules and ions, which was not conducive to form stable chemical bonds, and therefore led to the decrease of the adsorption efficiency ultimately.

The influence of temperature on removal efficiency of $\mathrm{Cu}^{2+}$ is revealed in Fig.7. The adsorption temperature 
experiments were performed in the range of $10 \sim 35^{\circ} \mathrm{C}$. The adsorption efficiency of $\mathrm{Cu}^{2+}$ increased slowly with the increase of temperature. When the temperature was at room temperature $\left(25^{\circ} \mathrm{C}\right)$, the adsorption efficiency of oxidized cellulose was highest (more than 99\%), Then it slightly reduced with the increase of temperature. In general, the impact of temperature on the adsorption efficiency of $\mathrm{Cu}^{2+}$ with NOCs as the adsorbent was not obvious, and the adsorption efficiency of $\mathrm{Cu}^{2+}$ was above $98 \%$ in a wide range $\left(10 \sim 30^{\circ} \mathrm{C}\right)$. Therefore it was sufficient to indicate that there would be a wide range of available temperature for the sorbent of oxidized cellulose in the wastewater treatment process.

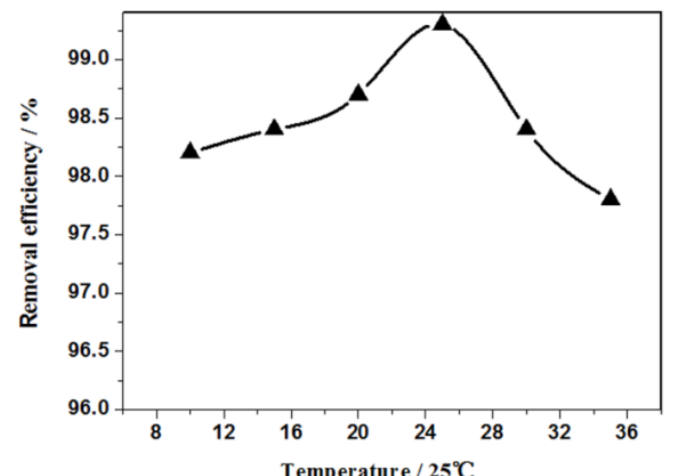

Figure 7.The influence of temperature on removal efficiency of $\mathrm{Cu}^{2+}$. 3.3.5. Effect of $p H$

The $\mathrm{pH}$ value of solution was another important factor to affect the adsorption process. Considering that the $\mathrm{pH}$ value of wastewater is usually below 7 , the simulated wastewater was performed in acid solution $(\mathrm{pH}$ ranged from 2 to 7) in our experiment. The influence of $\mathrm{pH}$ value on the removal efficiency of $\mathrm{Cu}^{2+}$ is shown in Fig.8. The adsorption efficiency was low at low $\mathrm{pH}$, and improved obviously with $\mathrm{pH}$ increased. The efficiency reached more than $96 \%$ when the $\mathrm{pH}$ ranged from 4 to 7 . Especially, under the $\mathrm{pH}$ value of 5 6, the highest removal efficiency was achieved, close to $99 \%$. The removal efficiency then began to decrease with increasing $\mathrm{pH}$. It can be expected that the removal efficiency would be smaller under $\mathrm{pH}>7$, because $\mathrm{Cu}^{2+}$ and $\mathrm{OH}^{-}$can easily form precipitation in the alkali solution.

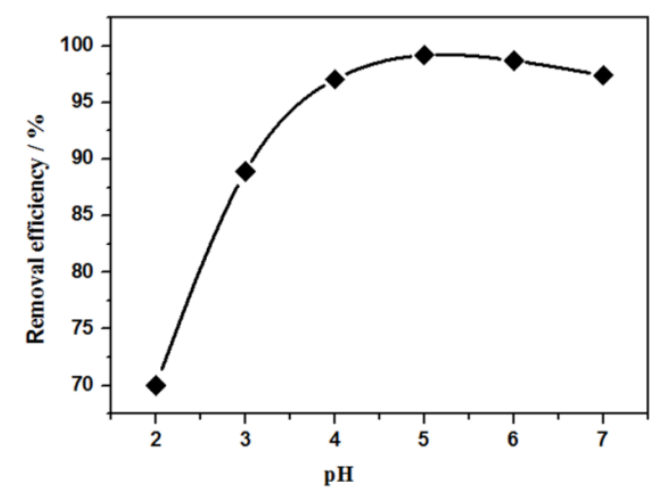

Figure 8.The influence of $\mathrm{pH}$ value on removal efficiency of $\mathrm{Cu}^{2+}$.

When $\mathrm{pH}$ was low, both effects of $\mathrm{pH}$ were disadvantageous to the efficiency. On the one hand, more hydronium ions were in the solution, which would compete with $\mathrm{Cu}^{2+}$ at the active site on the surface of the adsorbent; on the other hand, the adsorbent was in cationic atmosphere, this would decrease the number of the active site at which adsorption may take place. When $\mathrm{pH}$ was high, two competing factors determined the efficiency. $\mathrm{Cu}^{2+}$ and $\mathrm{OH}^{-}$formed deposits more easily, and the metal ions were surrounded by anions and not easily combined with adsorbent. At the same time, $\mathrm{pH}$ could also affect the acid-base properties of adsorbent, which led to a change of its structure. Therefore, there was an optimal $\mathrm{pH}$ for the efficiency. The condition of partial acid was more desirable to the copper removal especially when the $\mathrm{pH}$ in $5 \sim 6$.

\subsubsection{Adsorption isothermal}

The capacity of the adsorbent can be estimated through Adsorption equilibrium studies. It is usually described by adsorption isotherms. In order to gain an insight into the adsorption kinetics involved in the adsorption of $\mathrm{Cu}^{2+}$ ions by NOCs, The isotherm experiments were carried on the conditions of temperatures of $25^{\circ} \mathrm{C}$, the contact time of 1 $\mathrm{h}$ and the $\mathrm{pH}$ values of 5.5. And the experimental data were applied to the Langmiur and the Freundlich isotherm equations, respectively. Two equations $(3,4)$ are obtained as follows:

$$
\begin{array}{ll}
\frac{1}{q_{e}}=\frac{0.00875}{C_{e}}+0.00367 & \left(\mathrm{R}^{2}=0.9996\right) \\
\ln q_{e}=0.9520 \ln C_{e}-1.9944 & \left.\mathrm{R}^{2}=0.9992\right)
\end{array}
$$

Where $\mathrm{C}_{\mathrm{e}}\left(\mathrm{mg} \mathrm{l}^{-1}\right)$ is the equilibrium concentration of $\mathrm{Cu}^{2+}$ and $\mathrm{q}_{\mathrm{e}}\left(\mathrm{mg} \mathrm{g}^{-1}\right)$ is the adsorption capacity of $\mathrm{Cu}^{2+}$ at equilibrium.

According to the above equations, the high correlation coefficients indicate that the adsorption process of $\mathrm{Cu}^{2+}$ ions by NOCswas well corresponding with the Freundlichand Langmuir models. It also demonstrated that the adsorption process is a physical-chemical mixed mode which needs further detailed study. We compared simultaneously the adsorption capacities of NOCs with the cellulose and other various absorbents. Under the same conditions, the removal efficiency of $\mathrm{Cu}^{2+}$ by NOC and cellulose was $98.48 \%$ and $28.16 \%$, respectively. In conclusion, NOCs have high efficiency for the removal of $\mathrm{Cu}^{2+}$ from the wastewater.

\section{CONCLUSIONS}

The natural oxidation of celluloses is a new type of adsorbent, which can be synthesized by natural cellulose in an oxidized condition. We found that the material had a great effect on the adsorption of $\mathrm{Cu}^{2+}$ from wastewater. The optimum conditions of adsorption from the experiments were obtained. The removal efficiency of $\mathrm{Cu}^{2+}$ could reach as high as $99 \%$ under the conditions where the temperature was $25^{\circ} \mathrm{C}$, the $\mathrm{Cu}^{2+}$ concentration was $100 \mathrm{mg} \cdot \mathrm{l}^{-1}$ and the value of $\mathrm{pH}$ was $5 \sim 6$ in solution, dosing $0.175 \mathrm{~g}$ of natural oxidized celluloses and adsorption time $60 \mathrm{~min}$. Kinetic studies also showed that the adsorption isotherm of $\mathrm{Cu}^{2+}$ was corresponding with the Freundlich and Langmuir model. Other adsorption mechanism such as adsorption thermodynamics will be further studied subsequently. In conclusion, the present work predicts that the new adsorption material will have wide-ranging application in the sequestration or removal of metal ions and many other industrial processes in the future owing to the advantages such as raw materials easily available with many sources, large adsorption capacity, easy operation and low cost. 


\section{ACKNOWLEDGMENTS}

The authors are grateful for the support of National Natural Science Foundation of China (No. 51203083; 51303089) and Special Fund for Self-directed Innovation of Shandong Province of China (No. 2013CXB80201).

\section{REFERENCES}

[1] $\mathrm{Xu}, \mathrm{H} ., \mathrm{Liu}, \mathrm{Y} . . \mathrm{Mechanisms}$ of $\mathrm{Cd}^{2+}, \mathrm{Cu}^{2+}$ and $\mathrm{Ni}^{2+}$ biosorption by aerobic granules,Separation and Purification Technology, 2008, 58 (3), 400,(in Chinese).

[2] T. A.Kravchenko, L. L.Polyanskiy, V. A.Krysanov,et al..Chemical precipitation of copper from copper-zinc solutions onto selective sorbents, Hydrometallurgy,2009, 95, (1-2), 141.

[3] Xiong, Y. Y., Fu, Z. T.,Huang, W. S..Research on Treatment of Simulated Wastewater Containing Copper by Chemical Sedimentation Method, Environmental Protection Science,2013, 40(2), 35, (in Chinese)

[4] G.Ferraiolo, M.Zilli, A.Converti.Fly-ash disposal and utilization, J CHEM TECHNOL BIOT, 1990, 47, 281.

[5] V.K.Gupta, P.Singh, andN.Rahman.Adsorption behavior of $\mathrm{Hg}$ (II), $\mathrm{Pb}$ (II) and $\mathrm{Cd}$ (II) from aqueous solution on duolite C-433: a synthetic resin, J Colloid Interf. Sci., 2004, 275, 398.

[6] V. K.Gupta, V.K.Saini, N.Jain.Adsorption of As (III) from aqueous solutions by iron-oxide coated sand, J Colloid Interf. Sci., $2005,288,55$

[7] B.Volesky. Detoxification of metal-bearing effluents: biosorption for the next century, Hydrometallurgy, 2001, 59, 203.

[8] DONG, B. H., WANG, S. M., XU, Z. X.,et al.Study on adsorption properties of $\mathrm{Cu}^{2+}$ on nanometer-sized $\mathrm{SiO}_{2}$, Journal of Hubei University (JCR Science Edition), 2007, 29 (1), 60, (in Chinese).

[9] B. N.Estevinho, I.Martins, N.Ratola, et al.Removal of 2,4-dichlorophenol and pentachlorophenol from waters by sorption using coal fly ash from a Portuguese thermal power plant, J. Hazard. Mater.,2007, 143 (1-2), 535

[10] A. T.Paulino, L. B.Santos, J.Nozaki.Removal of $\mathrm{Pb}^{2+}, \mathrm{Cu}^{2+}$, and $\mathrm{Fe}^{3+}$ from battery manufacture wastewater by chitosan produced from silkworm chrysalides as a low-cost adsorbent, REACT FUNCT POLYM, 2008, 68(2), 634.

[11] ZHANG, J. Y., PU, L. J..Adsorption properties of $\mathrm{Cu}^{2+}$ laden wastewater with wheat straw and characteristic of its kinetics, Journal of Lanzhou University of Science and Technology, 2011, 37(03), 65, (in Chinese).

[12] Isobe, N., Chen, X.X., Kim, U.J., et al.TEMPO-oxidized cellulose hydrogel as a high-capacity and reusable heavy metal ion adsorbent, J. Hazard. Mater., 2013, 260, 195.

[13] Klemm, D., Heublein, B., Fink, H. P.,et al.Cellulose: fascinating biopolymer and sustainable raw material, Angew. Chem. Int. Ed., $2005,44,3358$

[14] Leandro, V. A. G., Osvaldo, K. J., Rossimiriam, P. F. et al.Adsorption of $\mathrm{Cu}$ ( II ), $\mathrm{Cd}(\mathrm{II})$, and $\mathrm{Pb}$ (II) from aqueous single metal solutions by cellulose and mercerized cellulose chemically modified with cuccinic anhydride, Bioresour. Technol., 2008, 99(8), 3077.

[15] David, W. O., Colin, B. and Thomas, F. O..Heavy metal adsorbents prepared from the modification of cellulose: a review, Bioresour.Technol.,2008, 99, 6709.

[16] ISOGAI, A. and KATO, Y..Preparation of polyuronic acid from cellulose by TEMPO-mediated oxidation, CELLULOSE, 1998, $5(3), 153$

[17] Saito, T., Shibata, I., Isogai, A. et al. Distribution of carboxylate groups introduced into cotton linters by the TEMPO-mediated oxidation, Carbohyd. Polym.,2005, 61 (4), 414.

[18] Luan, Z. k., Tang, H. X., study on surface properties of ore tailing particles and adsorption of heavy mental ions II. Adsorption of heavy metals onto ore tailing particles, Environmental Chemistry, 1993, 12 (5), 356, (in Chinese). 